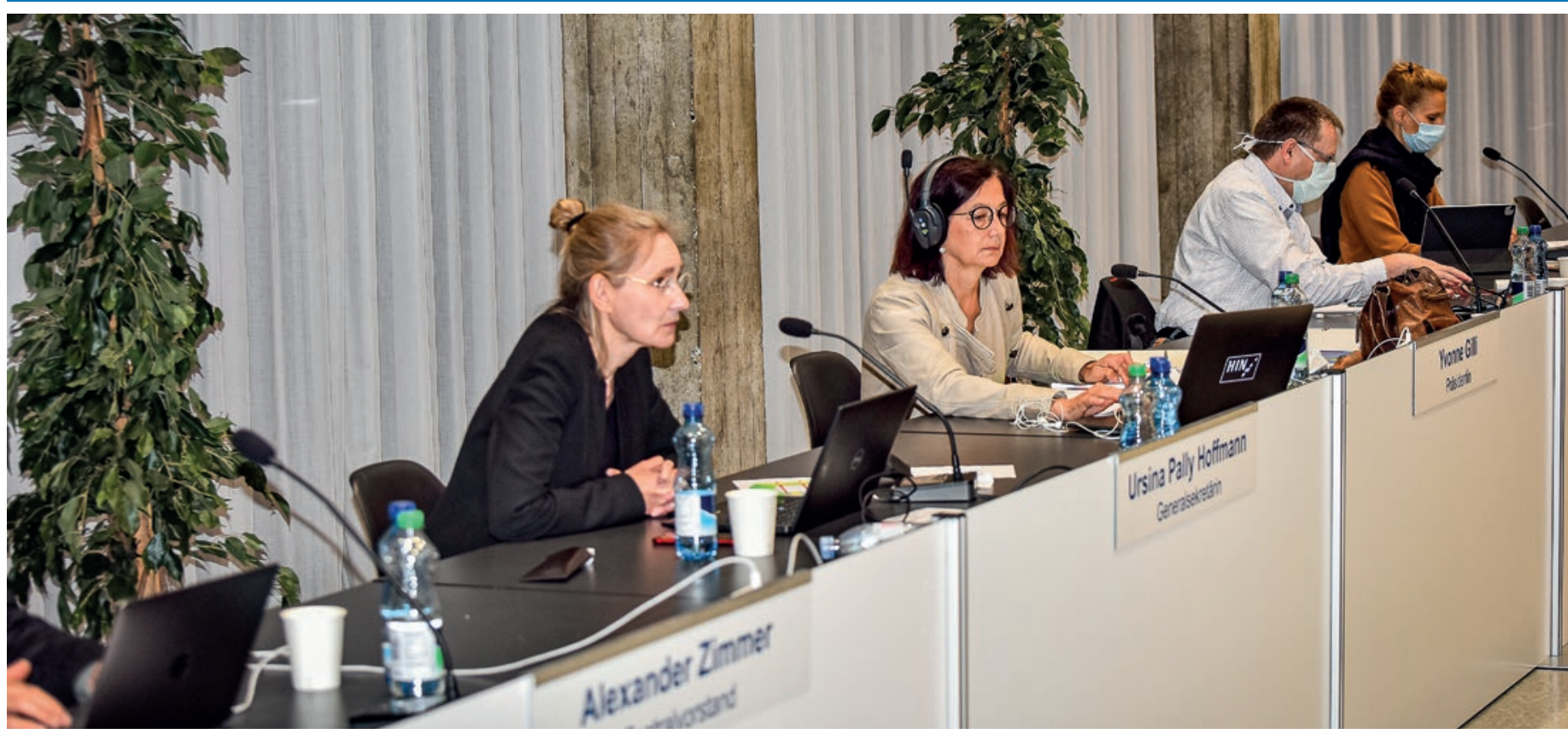

Corona oblige, la Chambre médicale se tient une nouvelle fois à distance. Seuls quelques collaborateurs et collaboratrices de la FMH, la secrétaire générale et les membres du Comité central sont sur place au Palais des Congrès de Bienne.

\title{
Procès-verbal décisionnel de la première Chambre médicale 2021
}

\section{Nicole Furgler}

Cheffe de la division Secrétariat central

\section{Jeudi 27 mai 2021}

\section{Message de bienvenue et informations}

Le quorum étant atteint, la Dre Yvonne Gilli / présidente de la FMH ouvre la séance virtuelle de la Chambre médicale du printemps 2021, retransmise depuis le Palais des Congrès à Bienne, et souhaite la bienvenue à tout le monde.

La Dre iur. Ursina Pally Hofmann / secrétaire générale de la FMH et cheffe de la division Service juridique rappelle que les délégué-e-s ont reçu le mercredi 26 mai 2021 les instructions techniques concernant le login, le contrôle d'accès à la séance et d'autres informations. Les délégué-e-s ayant le droit de discussion et de proposition et les personnes sans droit de vote ni d'éligibilité participent à la séance en streaming. Les prises de parole sont adressées par mail à ak[at]fmh.ch et sont ensuite lues. Ursina Pally Hofmann / secrétaire générale de la FMH et cheffe de la division Service juridique ex- pose la procédure en cas de problèmes techniques et renvoie aux différentes lignes d'assistance mises en place. Les personnes sur place doivent se conformer au plan de protection.

Ursina Pally Hofmann / secrétaire générale de la FMH et cheffe de la division Service juridique poursuit avec les autres informations organisationnelles habituelles et précise que tous les documents de séance supplémentaires normalement remis sous forme de dossier sont disponibles sur myFMH.

Yvonne Gilli / présidente de la FMH note la présence sur place de tous les membres du Comité central et de la secrétaire générale, à l'exception du Dr Michel Matter / membre du Comité central et responsable du département Prestations et développement professionnel qui s'est excusé.

La séance commence par un vote test suivi de la désignation du bureau. Celui-ci est composé de la présidente, du vice-président et de la secrétaire générale. Julien Duruz / Service juridique de la FMH et Dominik 


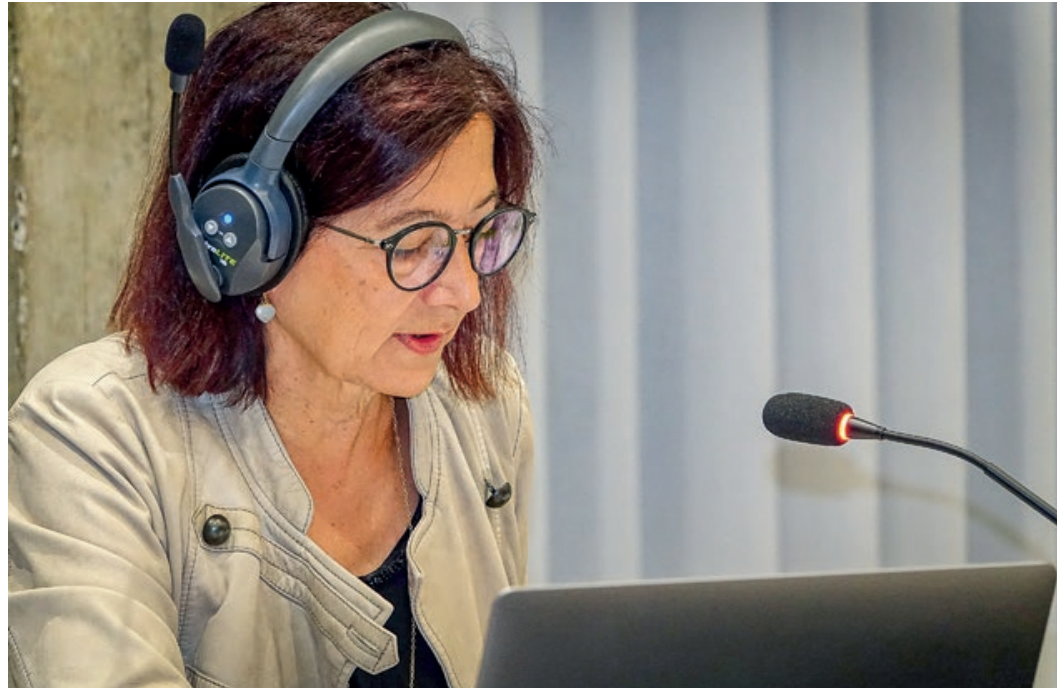

La présidente de la FMH, Yvonne Gilli, dirige la séance.

Kreuter / chef de la division ICT forment la codirection du bureau; Anne-Sylvie Thiébaud / cheffe de la Commission de déontologie de la FMH en assure la suppléance.

\section{Personnes invitées}

La présidente souhaite la bienvenue aux personnes invitées suivantes: Nicole Beutler (furrerhugi AG), Joachim Eder (président du conseil d'administration d'atstms), Stephanie Hauser (présidente de la swimsa), Markus Lehmann (directeur de FMH Consulting Services SA) et Damian Müller (conseiller aux États, canton de Lucerne).

Elle salue les personnes suivantes pour deux points à l'ordre du jour: Fabian Mollet de l'organe de révision BDO et German Clénin qui dépose une proposition en faveur de l'affiliation d'une nouvelle organisation. Jean Pierre Keller, vice-président de l'ISFM, et René Häller, FMH Consulting Services SA, sont tous les deux excusés.

\section{Scrutateurs}

Yvonne Gilli / présidente de la FMH propose le Dr Rudolf Ott, Commission de déontologie de la Société des médecins du canton de Bâle-Campagne, et la Dre iur. Caroline Hartmann, Service juridique de la FMH, comme scrutateurs.

\section{Proposition}

Approbation des scrutateurs

Décision

Les scrutateurs sont élus par 153 oui, 1 non et 2 abstentions.

Ordre du jour

Proposition

Approbation de l'ordre du jour

Décision
L'ordre du jour est approuvé par 156 oui, 0 non et 0 abstention.

Selon l'art. 11, al. 3, du Règlement d'exécution (RE), la Chambre médicale fixe au début de chaque séance l'heure limite à partir de laquelle elle ne peut plus prendre de décision ni procéder à des élections. La présidente propose de clore la séance à $17 \mathrm{~h} 30$.

Proposition pour l'heure de fin de la séance

La fin de la séance est fixée à 17h30. Au-delà de cette heure, il ne sera plus possible de prendre des décisions ou de procéder à des élections.

\section{Décision}

La proposition est adoptée par 155 oui, 3 non et 4 abstentions.

\section{Motion d'ordre: limitation du temps de parole}

Pour traiter les objets portés à l'ordre du jour de la Chambre médicale de ce jour, chaque personne a droit à un temps de parole de deux minutes. Les président-e-s des sociétés médicales avec droit de discussion et de proposition, ou leurs suppléant-e-s, ainsi que les porte-paroles du Comité central ne sont pas soumis à cette limitation du temps de parole.

\section{Décision}

La proposition est adoptée par 148 oui, 9 non et 2 abstentions.

Yvonne Gilli / présidente de la FMH rappelle que les propositions doivent être présentées par écrit, soit avant la séance, soit pendant la séance, à l'adresse électronique antrag[at]fmh.ch et que selon l'art. 11, al. 5, RE, les délégué-e-s, les membres du Comité central et la secrétaire générale ont chacun-e le droit de faire des propositions quant aux objets de l'ordre du jour soumis à la discussion.

\subsection{Discours d'ouverture de la présidente}

Yvonne Gilli / présidente de la FMH présente les défis que devra relever la FMH tant dans le domaine de la santé - par sa contribution à la maîtrise de la pandémie - que de la politique. Elle s'exprime tour à tour en allemand, français et italien.

La pandémie de Covid-19 occupe les autorités 24 heures sur 24, à commencer par les autorités de santé, et les met face à d'importants défis. C'est actuellement la campagne de vaccination qui est au premier plan des préoccupations de la FMH. Par rapport à nos voisins ou à Israël, nous accusons un certain retard. Tant la disponibilité des doses que leur distribution sont insatisfaisantes, et le jeu des tarifs ne couvrant pas les coûts, imposés par les autorités, n'est pas près de s'arrêter. On voit hélas ce que l'on peut attendre des tarifs étatiques: 
choix arbitraire, rémunération différente pour des prestations identiques, faux incitatifs et paralysie du système de santé, avec, pour résultat, un certain nombre de médecins de famille qui sont empêchés de participer à la campagne de vaccination. Cela ne fait que confirmer la nécessité pour la FMH de continuer à s'engager pour des tarifs négociés en connaissance de cause dans le cadre d'un partenariat éclairé.

Pour ce qui est du TARDOC, le projet est en bonne voie. Nous avons rempli toutes les conditions fixées par l'OFSP et effectué les adaptations exigées. Le tarif est en consultation au sein des offices et rien ne semble plus, sur le plan pratique, s'opposer à son approbation. Seulement voilà, la question revêt un caractère politique, raison pour laquelle il est si important que nous restions unis pour défendre ce tarif.

La FMH a intensifié sa présence au Parlement et sa collaboration avec l'administration. Les contacts avec la politique sont appelés à devenir toujours plus importants.

- Le $1^{\text {er }}$ volet de mesures destinées à freiner la hausse des coûts prévoit que les partenaires tarifaires prennent des mesures visant à piloter la fourniture de prestations. Dans le projet de loi, on peut lire qu'il s'agit de "prévoir des règles pour corriger toute hausse injustifiée des volumes et des coûts». Cela passerait par des adaptations tarifaires, des tarifs dégressifs, voire des remboursements. Dans les faits, cela reviendrait ni plus ni moins à fixer un plafond de coûts, car pour pouvoir sanctionner un accroissement injustifié, il faut bien déterminer jusqu'où ledit accroissement resterait «justifié». Sans compter que les partenaires tarifaires ne pourraient pas s'entendre librement entre eux, mais devraient prendre en compte les décisions des auto-

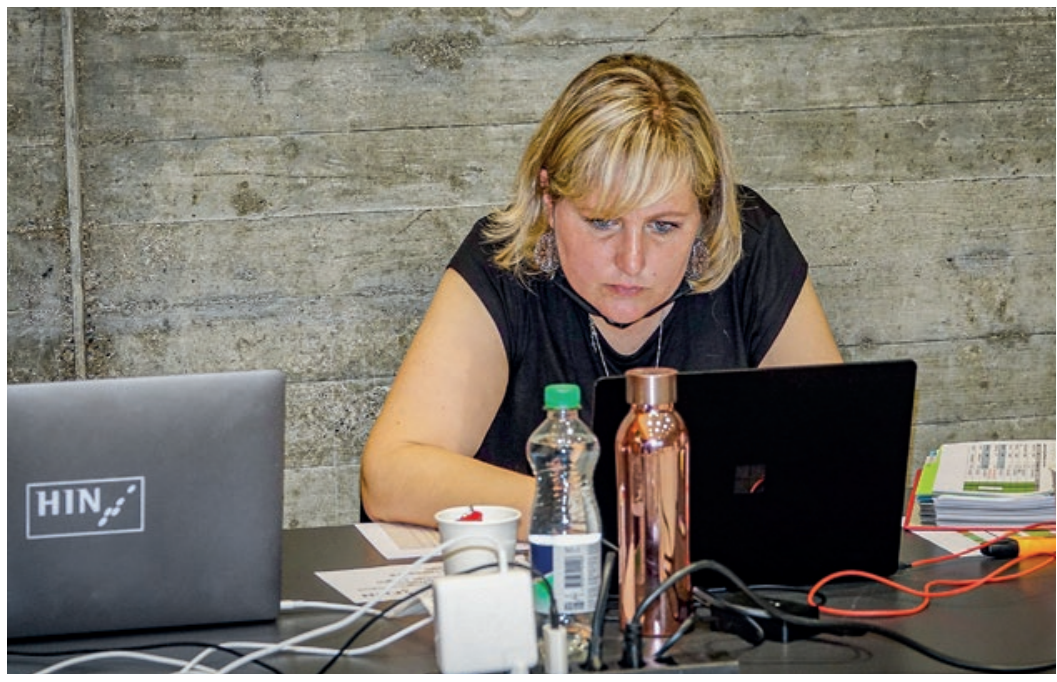

Claudia Marthaler, collaboratrice du Secrétariat central, contrôle l'accès à la séance virtuelle. rités aux fins de planification et de pilotage. Cela reviendrait à devoir budgéter nous-mêmes nos prestations sur mandat des autorités.

- Le $2^{\mathrm{e}}$ volet de mesures contient pour sa part un «objectif de coûts", ce qui revient de fait à fixer un budget global. L'objectif de coûts a entretemps été sorti du $2^{\mathrm{e}}$ volet de mesures et doit être proposé comme contre-projet indirect dans le cadre de la votation sur l'initiative du PDC «Baisser les primes - pour un frein aux coûts de la santé». Cela veut dire que si l'objet venait à être rejeté, on introduirait automatiquement les objectifs de coûts fixés par le Conseil fédéral. Ce projet de loi charge explicitement le Conseil fédéral de décider de combien de pour cent le coût global des prestations peut s'accroître chaque année, en lui donnant la possibilité de répartir les coûts sur plusieurs catégories de prestations. Si l'accroissement effectif des coûts excède ces limites, les partenaires tarifaires doivent adapter les conventions passées entre eux pour permettre l'introduction des mesures qui s'imposent pour abaisser les coûts.

Sur mandat de la FMH, le professeur Ueli Kieser a élaboré un avis de droit dans lequel il conclut à l'anticonstitutionnalité des mesures de pilotage des coûts prévues à l'art. 47c de la LAMal révisée et des objectifs de coûts. Une assurance se doit de définir par avance les risques couverts et les prestations, et ne peut pas les restreindre en imposant des plafonds de coûts. Les tarifs doivent être appropriés et répondre aux règles de l'économie d'entreprise. Ils ne sauraient être «corrigés» en vertu d'un objectif de coûts non atteint.

Avec ces projets de loi, on peut considérer que la FMH est mise au défi de repousser les assauts menés contre les fondements mêmes de notre système de santé. Dans la situation actuelle, il revient en particulier aux sociétés cantonales de médecine de rechercher le dialogue avec les directions de la santé publique de leur canton afin de les sensibiliser au problème de la constitutionnalité des mesures. Le Secrétariat général est à disposition pour leur envoyer la documentation nécessaire. Nous devons également nous préparer en vue du premier référendum, et donc à l'éventualité de voir introduits des tarifs dégressifs. Nous allons prochainement contacter les sociétés cantonales pour qu'elles puissent démarrer les préparatifs formels.

Il est très important que nous parlions d'une même voix, et nous prévoyons donc de garder régulièrement le contact avec nos membres. Nous nous sommes entretenus à cet effet avec les représentants du VEDAG, de la SMSR et de l'OMCT, en mars dernier, un échange qui s'est révélé précieux en vue d'optimiser et d'élargir 


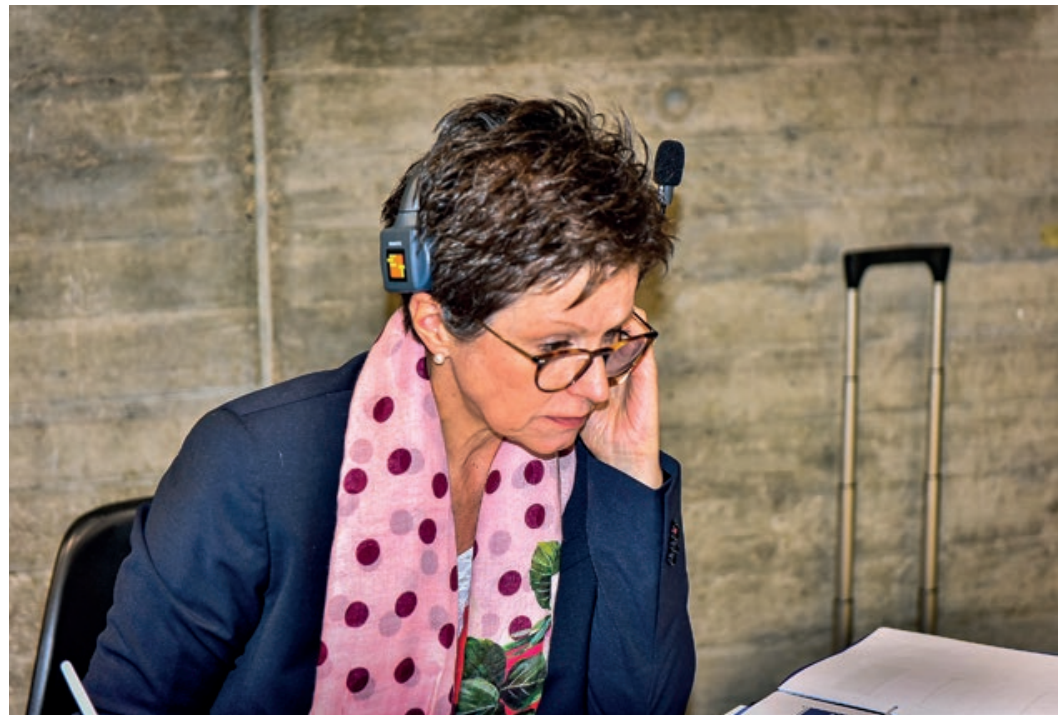

Nicole Furgler, cheffe de la division Secrétariat central, gère la séance (hôte) et la régie.

notre collaboration, étant donné le rôle clef dévolu aux sociétés cantonales dans la communication verticale. Pour ce qui concerne le $2^{\mathrm{e}}$ volet de mesures, Yvonne Gilli / présidente de la FMH explique qu'en instituant des points de premier contact, le Conseil fédéral souhaite non seulement pouvoir influer sur la façon dont les patients consultent leur médecin, mais être seul à décider de la rémunération desdits points de contact. Outre les volets de mesures visant à maîtriser les coûts, plusieurs dossiers sollicitent l'attention de la FMH:

- EFAS: le financement uniforme des prestations ambulatoires et hospitalières représente une opportunité à ne pas manquer pour notre système de santé.

- Article autorisant les projets pilotes: pourra-t-on nous obliger à mener des projets pilotes? Et ces projets pilotes devront-ils porter exclusivement sur la maîtrise des coûts?

- Et beaucoup d'autres: forfaits ambulatoires, dons d'organes, loi sur les professions de la psychologie, prévention du tabagisme, initiative «Pour des soins infirmiers forts", DEP, etc.

Sur la base d'un tableau chronologique, Yvonne Gilli / présidente de la FMH présente le calendrier prévu concernant les mesures de maîtrise des coûts et le budget global.

Yvonne Gilli / présidente de la FMH prend position sur les articles de presse parus ces derniers mois, qui entendent faire porter le chapeau au corps médical et tentent d'attiser les conflits, et sur la reprise encore plus polémique de ces articles dans les médias sociaux. La FMH veillera désormais à réagir publiquement à ce type d'attaques par une analyse approfondie des problématiques abordées.
Concernant les débats politiques à venir, la FMH a d'ores et déjà mandaté des analyses visant à déterminer les points forts et les points faibles de la profession en termes de réputation. Les trois quarts ou presque de la population estiment que les médecins défendent les intérêts des patients. Cette confiance de la part de notre patientèle revêt pour nous une importance capitale, surtout lorsqu'il s'agit de convaincre au Parlement ou de gagner lors de votations. Dans le même temps, $60 \%$ d'entre eux nous voient comme l'une des causes de la hausse des coûts de la santé. Nous devons agir avec doigté sur ce point, tout particulièrement dans le cadre des débats sur la rémunération des prestations médicales. Nos atouts sont notre crédibilité pour ce qui est des questions de santé et notre engagement pour la défense des intérêts des patients, nos points faibles la perception du public quant à notre proximité à l'industrie pharmaceutique et à notre rôle dans l'augmentation des coûts de la santé.

Yvonne Gilli / présidente de la FMH conclut son introduction en évoquant le certificat Covid, avec lequel il y avait bon espoir de pouvoir entamer une nouvelle collaboration avec l'OFSP, celle-ci ayant été jusque-là plutôt difficile. Depuis le début de la pandémie, les médecins installés ont été insuffisamment impliqués dans la gestion de la crise. La communication a été menée à très court terme. Souvent, nous avons appris par le biais des médias que la stratégie de test avait été modifiée ou qu'une autre décision pourtant importante pour nous avait été prise. La Confédération nous a maintenant informés qu'elle ne souhaitait pas notre solution pour le certificat Covid mais allait poursuivre son propre projet, et qu'il ne devrait y avoir qu'un seul certificat, raison pour laquelle nous devions arrêter sans délai le développement de notre projet. Ce que nous avons fait tout en continuant de nous tenir prêts et à disposition. Nous nous engagerons dans l'intérêt des patients et des fournisseurs de prestations, en faisant en sorte que la solution finalement adoptée soit efficace et nécessite aussi peu de travail administratif que possible.

En conclusion, la présidente résume:

\section{- Nous nous trouvons face à d'immenses défis:}

La pandémie, les volets de mesures visant à freiner la hausse des coûts, le budget global.

- Nous sommes sur le bon chemin

Le tarif, qui démontre notre capacité à trouver des compromis; une présence accrue dans les médias en tant que partenaire constructif.

- La mobilisation de toutes les organisations médicales est nécessaire:

Parler d'une seule voix, exploiter les synergies, trouver des ententes. 


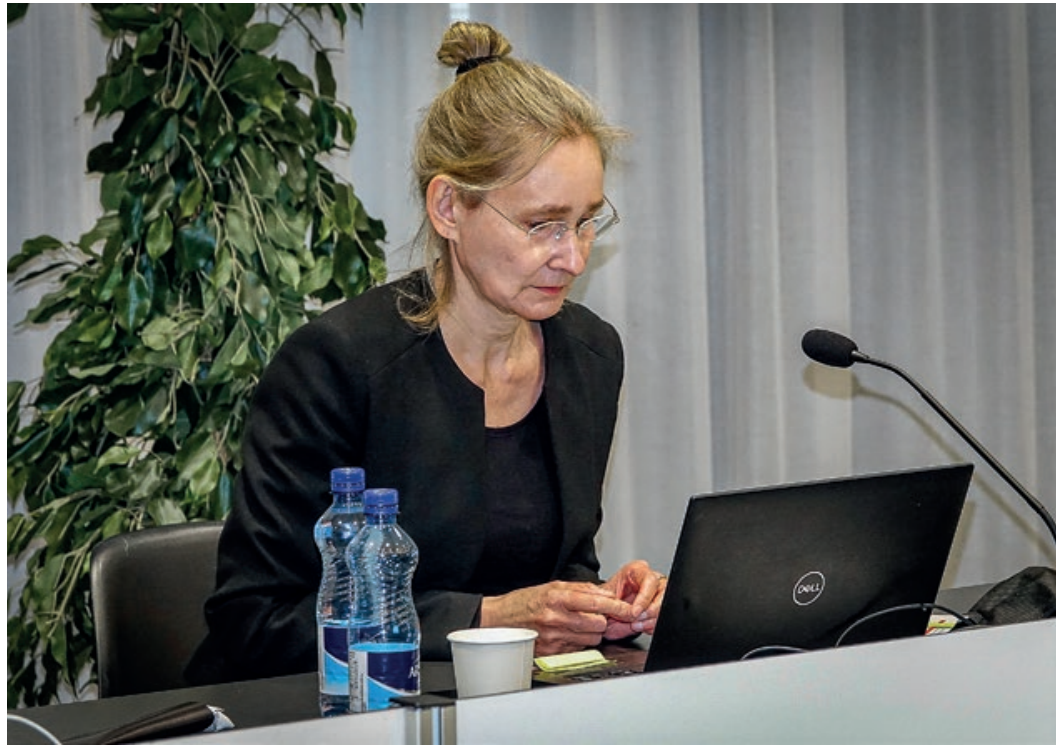

Ursina Pally Hofmann, secrétaire générale de la FMH et responsable de la division Service juridique. membres de la commission chargée des EPA. Elle souligne que cet énorme projet s'étendra sur plusieurs années et qu'il pourra être mené à bien et réussir seulement si un changement de perspective se met en place et si l'ensemble du corps médical est prêt à porter ce changement de culture dans la manière de former les futurs médecins.

Christoph Hänggeli / directeur de l'ISFM poursuit avec les points suivants:

L'année écoulée a été marquée par le Covid-19. De nombreux congrès et cours de formation postgraduée ont été annulés, les examens de spécialiste ont dû être ajournés et des réductions de temps de travail prononcées dans les établissements de formation. Une réglementation extraordinaire a dû être mise en place dans presque chaque discipline, pour que les candidat-e-s puissent obtenir leur titre de manière juridiquement valable. Tout cela a engendré une énorme charge de travail supplémentaire pour les collaboratrices de l'ISFM et des retards dans le traitement des dossiers. Mais en fin d'année, le nombre de titres de spécialiste octroyés a quand même atteint le chiffre record de 1700. Comme l'offre de formation continue a elle aussi été massivement réduite, l'ISFM a ajouté un crédit Covid à hauteur de 35 crédits pour l'année 2020 et de 25 pour 2021 à chaque profil de la plate-forme de formation continue.

Des réglementations spécifiques ont également été créées pour les programmes de formation complémentaire et les formations approfondies interdisciplinaires, principalement en ce qui concerne les recertifications.

Un des effets collatéraux de ces surcharges d'activité a également été les retards pris dans l'introduction du nouveau logbook électronique.

Christoph Hänggeli / directeur de l'ISFM évoque les principaux chiffres clés et développements:

L'ISFM a octroyé près de 400 titres de plus que l'année précédente. $44 \%$ des titres de spécialiste délivrés l'ont été à des médecins venus de l'étranger, dont $45 \%$ d'Allemagne. Le rapport entre titres fédéraux octroyés et titres étrangers reconnus est énorme dans certaines disciplines, notamment en chirurgie cardiaque, où l'on compte quatre fois plus de titres de spécialiste étrangers reconnus que de titres fédéraux octroyés. En chirurgie plastique, ce rapport est de un sur trois.

Le MedReg fait état de 1400 enregistrements de diplômes de médecin étrangers non reconnus (ce qu'on appelle les diplômes de pays tiers).

Toutes les informations détaillées du rapport annuel peuvent être consultées en ligne sur le site www.siwf.ch. Proposition

La Chambre médicale décide d'approuver le rapport annuel 2020 de l'ISFM. 


\section{Décision}

La proposition est acceptée par 153 oui, 1 non et 2 abstentions.

\subsection{Rapport annuel 2020 de la FMH}

Ursina Pally Hofmann / secrétaire générale de la FMH et cheffe de la division Service juridique montre le rapport annuel 2020 de la FMH, publié le 21 avril 2021, qui peut être consulté sur le site de la FMH: www.fmh.ch. L'année 2020 a été nettement marquée par le Covid-19, ce dont fait état le rapport annuel non sans oublier les nombreuses autres activités qui ont jalonné l'année. Le point "Chronologie» passe en revue les principaux événements de l'année. Ursina Pally Hofmann / secrétaire générale de la FMH et cheffe de la division Service juridique montre ensuite le film sur la statistique médicale et indique les thèmes traités par le rapport annuel et le rapport de situation.

Ursina Pally Hofmann / secrétaire générale de la FMH et cheffe de la division Service juridique invite l'auditoire à lire le rapport annuel en détail.

\section{Proposition}

Le Comité central de la FMH propose à la Chambre médicale d'approuver le rapport annuel 2020 de la FMH.

Décision

La proposition est adoptée par 150 oui, 0 non et 2 abstentions.

\section{Comptes annuels 2020}

\subsection{Compte de résultat 2020 de l'ISFM}

Christoph Hänggeli / directeur de l'ISFM détaille les comptes 2020, qui font état d'un bénéfice de 994000 francs (budgétisé à 112000 francs). Ce bon résultat est lié principalement au nombre élevé et inattendu de titres décernés (plus de 350 titres de spécialiste en plus par rapport à l'année précédente).

Quelques écarts importants par rapport au budget méritent d'être mentionnés:

Recettes (écart de +1100000 francs par rapport au budget): moins de rentrées liées aux visites d'établissements contre des recettes supplémentaires pour les titres de spécialiste, formations approfondies, médecin praticien, plate-forme de formation continue, certification d'établissements de formation postgraduée.

Dépenses (écart de +208000 francs par rapport au budget): davantage de dépenses liées aux réserves contre une baisse des dépenses pour le personnel, la promotion de projets, la plate-forme de formation continue et les facturations internes.

Le niveau de fortune au $1^{\text {er }}$ janvier 2021 s'élève à 3480000 francs.

\subsection{Compte de résultat 2020 de la FMH}

Le compte de résultat 2020 de la FMH se solde par un bénéfice à hauteur de 1,466 million (1,919 million l'année précédente). Une perte de 0,201 million avait été budgétisée.

Patrick Egger / chef de la division Finances et administration compare différentes positions du compte de résultat de la FMH à celles de l'année précédente.

Recettes: les cotisations de membres y compris les cotisations spéciales marquent une légère hausse en raison de l'accroissement du nombre de médecins en exercice. Les recettes pour prestations ont légèrement baissé. Les recettes résultant de projets proviennent principalement du projet «PEPra - la prévention fondée sur les preuves au cabinet médical», financé en majorité par la fondation Promotion Santé Suisse.

Dépenses: les dépenses directes ont légèrement diminué. La FMH a organisé moins de rencontres et manifestations, a payé moins de défraiements; les dépenses du Bureau d'expertises ont par ailleurs baissé tandis que celles pour les projets ont augmenté dans une moindre mesure. Les frais de personnel (salaires et honoraires de tiers) ont augmenté notamment en raison d'ajustements salariaux suite à des changements de fonction ou de rééquilibrage de salaire (le pourcentage de postes est pratiquement inchangé), des formations continues suivies et de dépenses plus élevées pour les honoraires de tiers.

La hausse marquée des autres charges d'exploitation est liée au déménagement dans les locaux intermé diaires, au loyer et à l'aménagement de ces locaux. Les dépenses pour la publicité ont également augmenté. La rénovation de l'Elfenstrasse 18 a commencé en 2020. Pour l'instant, peu d'investissements augmentant la valeur de l'immeuble ont été effectués car il s'agissait au début principalement de travaux de démolition. C'est pour cette raison que les amortissements n'ont que légèrement augmenté par rapport à l'année dernière.

Charge financière: la recomposition du portefeuille financier a conduit à une hausse des taxes. D'autres dépenses sont liées au fait que quelques titres ont été vendus en dessous de leur valeur comptable.

Produits financiers: les bénéfices réalisés sur les cours de différents titres ont permis d'importants gains financiers. La problématique des taux négatifs, à laquelle la FMH a réagi, a été la principale raison de recomposer le portefeuille.

Autres positions: le coût des activités annexes a aug menté surtout en raison des dépenses courantes de la rénovation du siège de l'Elfenstrasse 18. Les dépenses extraordinaires englobent la constitution de réserves pour les formations TARDOC et les amortissements ex- 


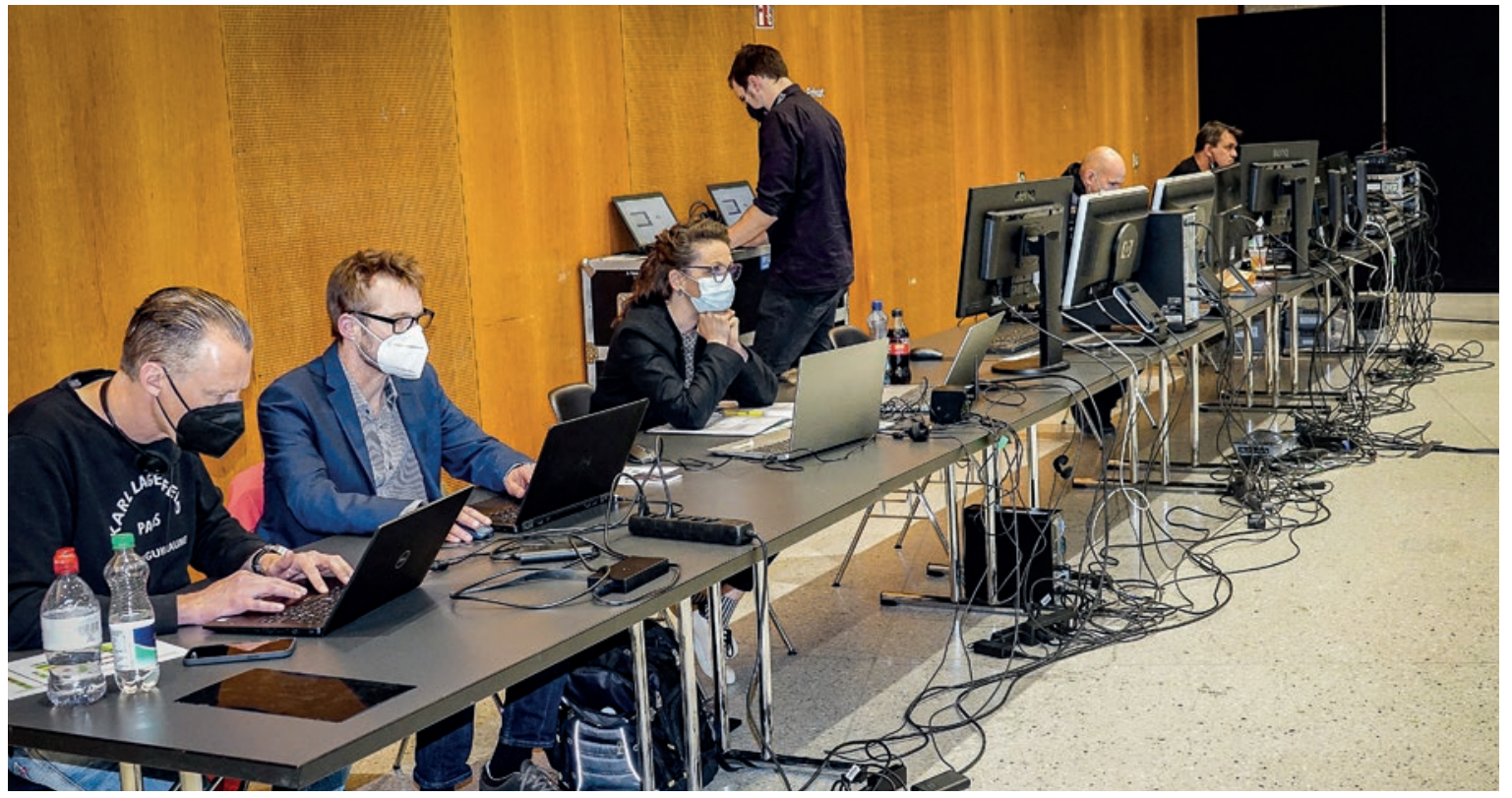

Les collaboratrices et collaborateurs de la FMH (de g. à d.: Dominik Kreuter, chef de la division ICT, Nils Graf, collaborateur du Service juridique, Natacha Sommariva, collaboratrice du Secrétariat central) et l'équipe technique externe veillent à la parfaite retransmission de la séance.

traordinaires concernant la participation à AD Swiss Net SA. Suite aux gains enregistrés ces deux dernières années par la FMH, une somme provisoire a été prévue pour l'impôt sur le bénéfice.

Performance des placements: le portefeuille de la FMH se compose de liquidités, d'obligations, d'actions, de fonds immobiliers, de métaux précieux et d'une participation à la Caisse des médecins. Il est très réjouissant que ce portefeuille ait atteint une performance de 1,98\% malgré le coronavirus. Les actions étrangères et le fonds immobilier en Suisse ont également eu un impact positif sur ce résultat.

\subsection{Comptes annuels 2020 de la FMH}

Les comptes annuels de la FMH se composent des comptes de la FMH et des comptes de l'ISFM, soit un bénéfice en 2020 de 2,460 millions (1,993 million l'année précédente). La somme au bilan s'élevait au 31 décembre 2020 à 38,161 millions.

Utilisation du résultat 2020: sur les 2,460 millions, 1,466 est attribué au capital de la FMH et 0,994 aux réserves de l'ISFM.

Fortune au $1^{\text {er }}$ janvier 2021: après l'attribution du bénéfice, la FMH fait état d'une fortune de 14,659 millions et l'ISFM, de 3,480 millions. Le taux de financement propre (capital propre par rapport à la somme au bilan) est de $47,5 \%$.

\subsection{Comptes consolidés 2020 de la FMH}

La FMH détient des participations dans six sociétés et les consolide selon les méthodes suivantes: HIN Health Info Net SA (consolidation globale), EMH SA (consolidation globale), ats-tms SA (consolidation proportionnelle), AD Swiss Net SA (méthode de mise en équivalence), SwissDRG SA (valeur d'acquisition) et NewIndex SA (valeur d'acquisition).

$\mathrm{Au} 31$ décembre 2020, le bilan présente des actifs circulants de 41,111 millions et des actifs immobilisés de 9,407 millions. Les passifs sont constitués de fonds étrangers à hauteur de 26,771 millions et de fonds propres à hauteur de 23,747 millions (somme au bilan: 50,518 millions, année précédente: 43,758 millions). Presque toutes les sociétés de participation (à l'exception de SwissDRG SA) ont terminé l'année par des bénéfices. Le résultat annuel se termine donc par un bénéfice de 2,838 millions (2,493 millions l'année précédente).

\subsection{Rapport de l'organe de révision}

Patrick Egger / chef de la division Finances et administration confirme l'examen des comptes annuels 2020 (FMH et ISFM) par l'organe de révision qui a analysé en détail le système de contrôle interne (concernant le personnel) et atteste à la FMH un niveau de conscience élevé en matière de contrôle. L'organe de révision émet les recommandations suivantes: la documentation relative au système de contrôle interne doit être finalisée; l'activation des coûts de construction doit être affinée à partir de 2021 et, si possible, mise en œuvre conformément à la comptabilité. Comme il n'y a pas de pertes à reporter et que les comptes font à nouveau état d'un bénéfice, il faut prévoir une somme provisoire de 260000 francs pour l'impôt sur le bénéfice. La participation à $\mathrm{AD}$ Swiss Net SA a donné lieu à une correction 


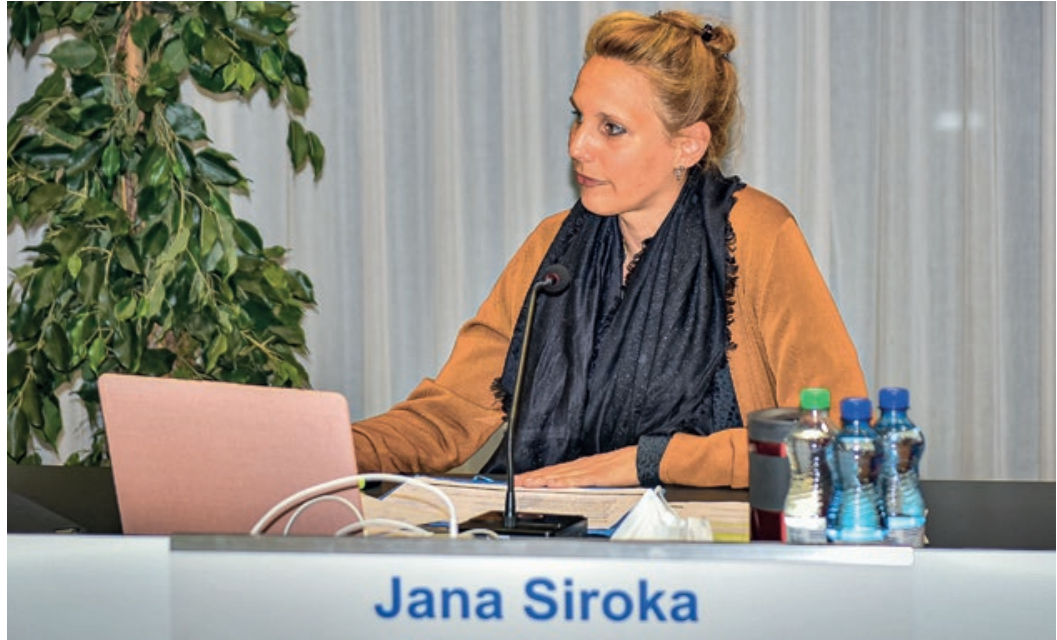

Jana Siroka, responsable du département Médecine et tarifs hospitaliers

exceptionnelle à 1.00 franc au 31 décembre 2020. La valeur nominale de participation s'élève à 40000 francs. Fabian Mollet / organe de révision $B D O$ confirme que les comptes sont conformes à la loi et aux Statuts et qu'il existe un système de contrôle interne relatif à l'établissement des présents comptes annuels. L'organe de révision recommande d'approuver les comptes annuels 2020 ainsi que les comptes consolidés 2020.

\subsection{Rapport de la Commission de gestion (CdG)} Philippe Vuillemin / président de la CdG commence par des informations relatives à la nouvelle composition de la CdG, ses tâches et ses méthodes de travail. Les points suivants ont fait l'objet d'une attention particulière:

- la politique salariale appliquée au Comité central

- le logbook électronique

- GEVER

- la communication de crise par la FMH, à tous les échelons

- les relations de la FMH avec les autorités fédérales et cantonales, mais aussi internationales

Concernant l'ISFM, il note que les relations administratives entre la FMH et l'ISFM se sont améliorées, d'où une meilleure efficience. Alors que l'ISFM est déjà indépendant de la FMH du point de vue opérationnel, le débat reste entier quant à la forme juridique future de l'ISFM. Les comptes et bilans respectifs de la FMH et de l'ISFM ont été présentés, examinés et validés lors des séances des 16 et 17 mars 2021. Philippe Vuillemin / président de la CdG les repasse en revue. Il termine par quelques remarques finales:

- Il faut garder une réserve pour l'action politique.

- Il faut garder la maîtrise des heures supplémentaires et de l'accroissement du personnel.

- Il faut réfléchir au sens des hauts salaires de la FMH sous l'angle de l'efficience, des contraintes médiatiques et politiques.
- Une réflexion sur les cotisations des membres doit être faite.

Au nom de la CdG, il recommande à la Chambre médicale d'approuver les comptes 2020 et remercie les présidentes de la FMH et de l'ISFM, la secrétaire générale de la FMH, les directeurs financiers et tous les cadres et le personnel de la FMH pour leur disponibilité.

Approbation des comptes annuels 2020 Proposition

Le Comité central de la FMH et respectivement la direction de l'ISFM proposent à la Chambre médicale d'approuver les comptes annuels 2020, et plus exactement:

- le compte de résultat 2020 de l'ISFM

- le compte de résultat 2020 de la FMH

- les comptes annuels 2020 de la FMH

- les comptes consolidés 2020 de la FMH

\section{Motion d'ordre de la présidente de la FMH}

Il est proposé à la Chambre médicale de se prononcer par un vote unique sur les comptes annuels 2020 (points 3.1 à 3.4).

Décision

La Chambre médicale décide par 149 oui, 4 non et 3 abstentions de se prononcer par un vote unique sur les comptes annuels 2020 (points 3.1 à 3.4).

Décision

Les comptes annuels 2020 de la FMH (points 3.1 à 3.4) sont acceptés par 145 oui, 3 non et 0 abstention.

\section{Octroi des décharges}

\subsection{Octroi de la décharge au Comité de l'ISFM Proposition de la direction de l'ISFM}

La Chambre médicale décide de suivre les recommandations de l'organe de contrôle et de la CdG et d'octroyer la décharge à la direction de l'ISFM pour l'exercice 2020.

Décision

La décision est acceptée par 149 oui, 0 non et 4 abstentions.

\subsection{Octroi de la décharge au Comité central de la FMH}

La Chambre médicale décide de suivre les recommandations de l'organe de contrôle et de la Commission de gestion et d'octroyer la décharge au Comité central de la FMH pour l'exercice 2020.

Décision

La décision est acceptée par 144 oui, 0 non et 3 abstentions. 


\section{5. Élections et élections de confirmation}

\subsection{Confirmation des déléguées et délégués nommés à l'Assemblée des délégués} Les organisations délégantes (CMPR, SFSM, SMSR, ASMAC) proposent à la Chambre médicale de confirmer les (nouveaux) membres de l'Assemblée des délégués de la FMH:

CMPR

actuelle

nouveau

JUNDT HERMAN Nicole, JENNY Philipp, Altstätten Étagnières

SFSM

actuelle

SCHWARZKOPF

nouveau

Ann Kathrin, Berne

SMSR

actuel

nouveau

GUSMINI Mauro Walter, BÜNZLI Dominique, Peseux La Chaux-de-Fonds

ASMAC

actuel

nouvelle

VACANT

WANG Anna, Schlieren

Décision

Les déléguées et délégués nommés à l'Assemblée des délégués sont confirmés par 143 oui, 1 non et 7 abstentions.

\subsection{Confirmation des déléguées et délégués} suppléants nommés à l'Assemblée des délégués Le CMPR, l'organisation délégante, propose à la Chambre médicale de confirmer le (nouveau) délégué suppléant à l’Assemblée des délégués de la FMH:

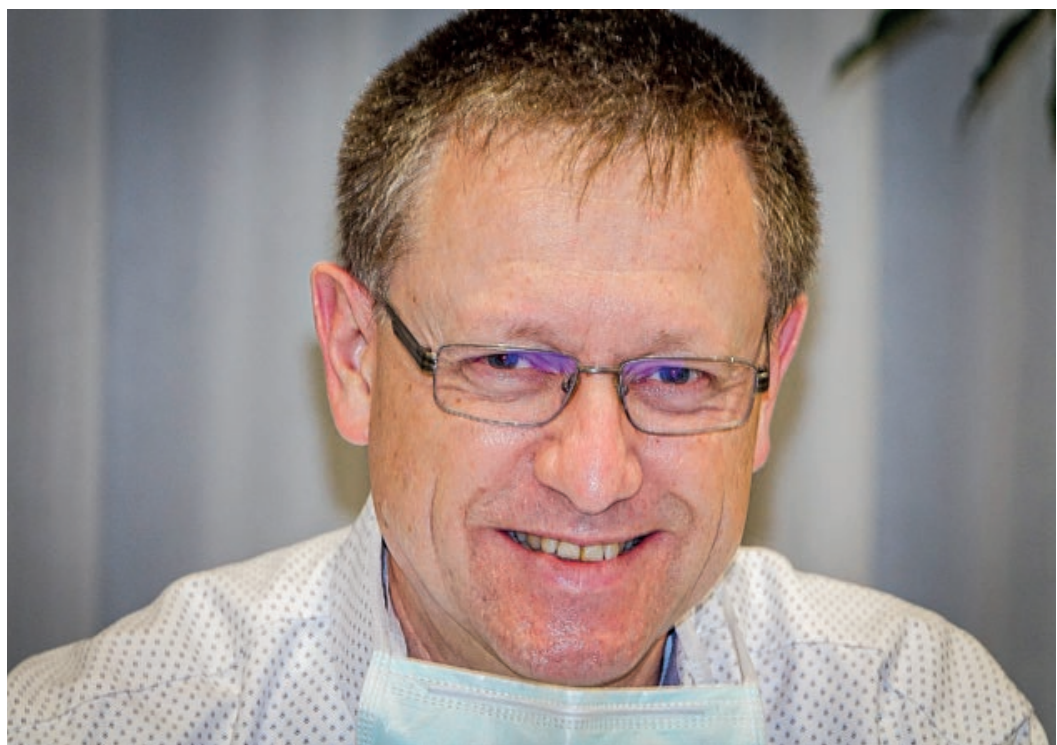

Christoph Bosshard, vice-président de la FMH et responsable du département Données, démographie et qualité.
CMPR

actuel

nouveau

VACANT RAMELLI Gian Paolo, Bellinzone

Décision

Le délégué suppléant nommé à l'Assemblée des délégués est confirmé par 147 oui, 0 non et 4 abstentions.

\section{Stratégie de la FMH, législature 2021-2024}

Yvonne Gilli / présidente de la FMH présente les enjeux et les objectifs de la FMH pour la législature 2021-2024.

- Les enjeux ont été identifiés par le biais d'un sondage auprès des membres de la FMH, des délégué-e-s à la Chambre médicale et d'expert-e-s tant internes qu'externes.

- Les objectifs stratégiques de la FMH ont été ventilés entre sept domaines (correspondant aux départements du Comité central) et un domaine Secrétariat général.

- En mars 2021, l’Assemblée des délégués a appuyé la stratégie et donné quelques idées en vue de sa mise en œuvre. Ces dernières ont concerné les thèmes eHealth, pilotage fondé sur des données et conditions-cadres dans les hôpitaux. De manière générale, la FMH doit agir de manière plus proactive et s'orienter davantage sur l'innovation.

- Comme déjà par le passé, les objectifs stratégiques de la FMH et les mesures définies dans le cadre de la mise en œuvre de la stratégie sont vérifiés une fois l'an par le Comité central et complétés au besoin.

Le processus stratégique se décline en quatre étapes:

- Enjeux

Quelles sont les exigences internes et externes? Perspective très large avec un horizon temporel éloigné.

- Objectifs

Quels objectifs et sous-objectifs s'agit-il d'atteindre?

- Mesures

Comment faire pour atteindre nos objectifs et nos sous-objectifs?

- Revue annuelle

Les enjeux d'hier sont-ils toujours d'actualité?

Où en sommes-nous dans la réalisation de nos objectifs?

Le sondage sur la stratégie et la pondération du Comité central a permis faire ressortir 14 enjeux centraux:

1. Politique suisse de la santé: représentation des intérêts

2. Tarifs ambulatoires et maintien de l'autonomie tarifaire

3. Conditions de travail et attrait de la profession 


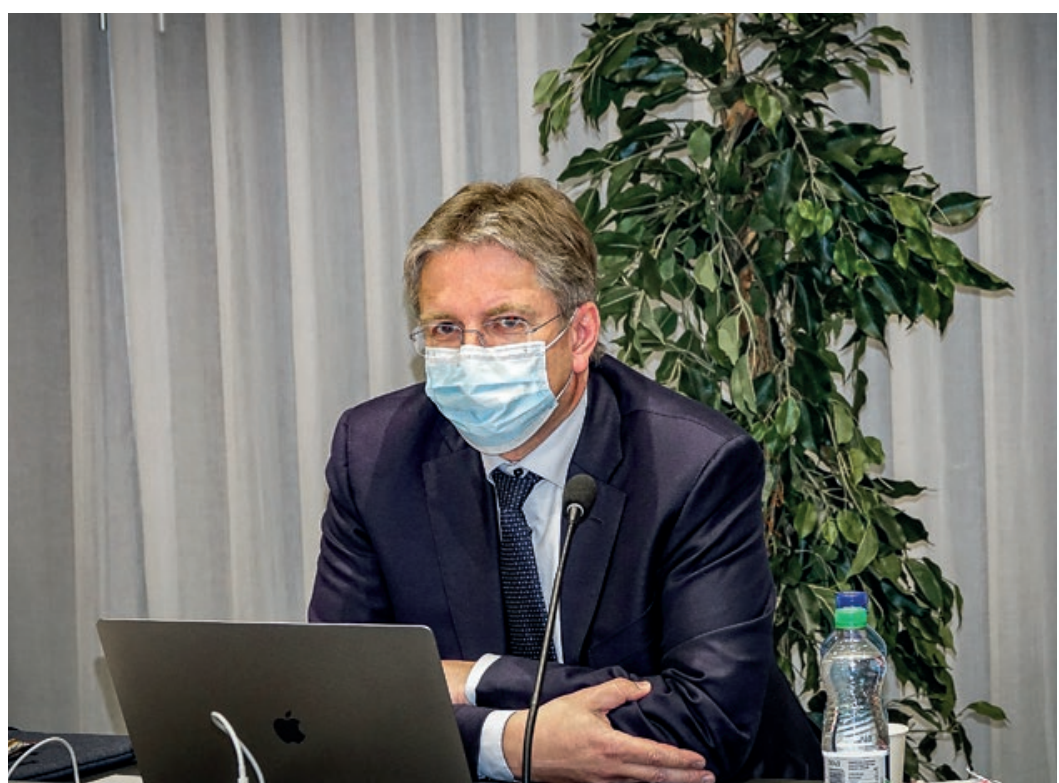

Carlos Quinto, responsable du département Santé publique/Professions de la santé.

4. Cohésion / présentation d'un front uni

5. Volume des tâches administratives

6. Pression temporelle sur la relation médecin-patient

7. Pression sur les coûts et prise en charge médicale

8. Pénurie de médecins et garantie de la prise en charge

9. Formation prégraduée, postgraduée et continue

10. Image du corps médical

11. Conciliation vie familiale / professionnelle

12. Admission / liberté de contracter / obligation de contracter

13. Représentation des positions de la FMH dans les médias

14. Santé des médecins et burn-out

La mission de la FMH

- En tant qu'organisation indépendante, la FMH défend les intérêts des médecins et les soutient dans l'exercice de leur profession.

- En tant que partenaire crédible, la FMH s'engage avec d'autres acteurs pour améliorer durablement le domaine de la santé.

- La FMH s'engage pour que tous les patients aient accès à des soins de haute qualité et financièrement abordables.

Objectifs principaux des départements et du Secrétariat général

1. La FMH œuvre pour un corps médical uni et s'engage pour des soins médicaux forts.

2. La FMH s'investit pour la transparence dans les activités qualité et pour le maintien de l'autonomie en matière de qualité en s'appuyant sur des données fiables.

3. La FMH façonne activement l'avenir de la profession médicale et fournit des prestations attractives à ses membres.

4. La FMH s'investit pour préserver l'autonomie tarifaire, pour assurer une rémunération adéquate et pour renforcer la prise en charge ambulatoire.

5. La FMH s'engage pour une rémunération adéquate et pour de bonnes conditions-cadres régissant l'activité médicale dans le secteur hospitalier.

6. La FMH s'investit pour une numérisation adaptée aux besoins de la prise en charge médicale et véritablement utile.

7. La FMH soutient et renforce la santé publique dans le sens souhaité par les médecins.

8. Le Secrétariat général administre, coordonne et développe la FMH en tant qu'organisation experte.

Les responsables des départements et la secrétaire générale détaillent les objectifs principaux et les sous-objectifs de leur département et du Secrétariat général. Ces objectifs sont déterminants pour la formulation des objectifs annuels et du budget de la FMH.

\section{Proposition}

La Chambre médicale approuve ces objectifs stratégiques pour la période législative 2021-2024.

Décision

La proposition est acceptée par 146 oui, 3 non et 5 abstentions.

\section{Modification des Statuts}

\subsection{Adaptation de l'annexe III:}

organisations médicales autorisées à intervenir, sans droit de vote

Yvonne Gilli / présidente de la FMH et le Dr German E. Clénin / président de la SEMS présentent la proposition d'adaptation de l'annexe III des Statuts.

La société médicale Sport \& Exercise Medicine Switzerland (SEMS), anciennement Société suisse de médecine du sport (SSMS/SGSM), a été fondée en 1949 et a pour objectif la promotion de la médecine du sport et des activités qui lui sont apparentées, dans le cadre pluridisciplinaire de la recherche, de l'enseignement et de la pratique. Elle se préoccupe de la santé de la population en général, de l'athlète d'élite aux sportifs handicapés, en passant par le sport populaire.

Elle a inscrit la collaboration avec la FMH dans ses Statuts, en particulier concernant la formation initiale, postgraduée et continue de ses membres. Elle délivre d'ailleurs un diplôme de formation approfondie inter- 
disciplinaire en médecine du sport et de l'exercice qui est reconnu par l'ISFM depuis 1999. En 2018, la SEMS a également participé à la révision de l'annexe 5 au Code de déontologie de la FMH (Directive pour la prise en charge médicale des sportifs).

Le nombre total de ses membres se montait, au début mars 2021, à 821 dont 608 sont des membres actifs (médecins en activité). Après vérification par le Secrétariat général de la FMH, une claire majorité des membres est affiliée à la FMH.

Le 4 février 2021, la SEMS a déposé une demande d'admission à la Chambre médicale, en tant qu'organisation de la FMH ayant droit de discussion et de proposition, mais sans droit de vote ni d'éligibilité, conformément à l'art. 25, al. 2, des Statuts de la FMH. Elle remplit les exigences fixées par cet article. Le Comité central et l'Assemblée des délégués soutiennent la demande d'admission de la SEMS.

Proposition

La Chambre médicale admet la SEMS en tant qu'organisation ayant droit de discussion et de proposition, mais sans droit de vote ni d'éligibilité, conformément à l'art. 25, al. 2, des Statuts de la FMH.

Décision

La proposition est acceptée par 133 oui, 6 non et 9 abstentions.

\section{Modifications du Règlement d'exécution}

Aucune proposition déposée.

\section{Modifications du Code de déontologie}

Aucune proposition déposée.

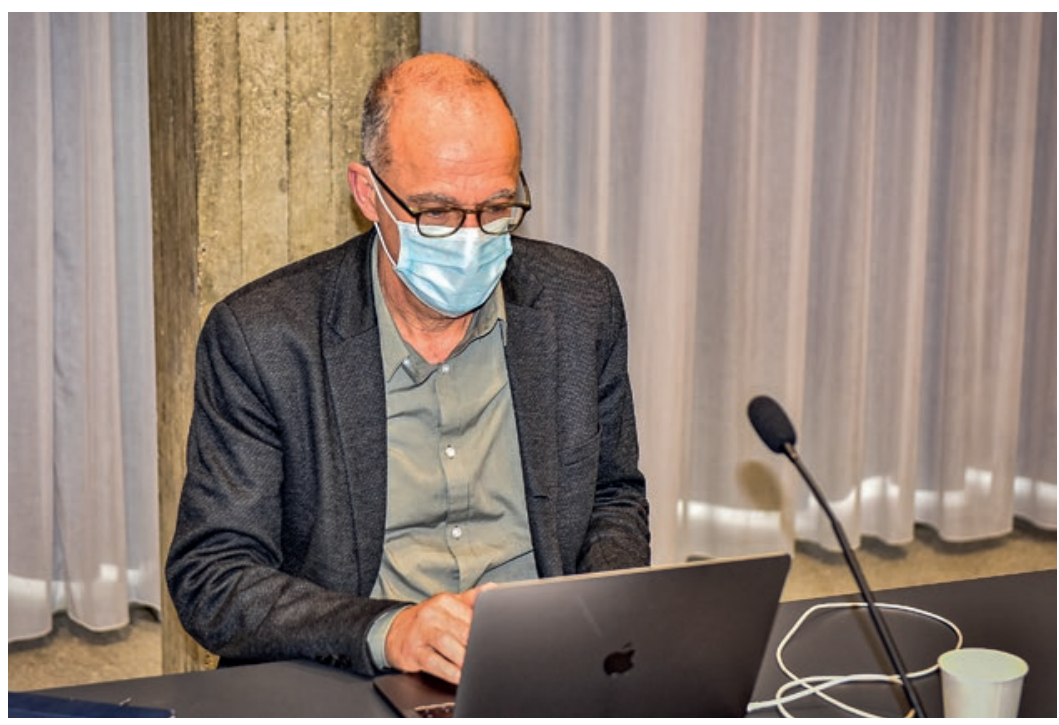

Alexander Zimmer, responsable du département Numérisation/eHealth.

\section{TARDOC: révision du tarif ambulatoire}

Urs Stoffel / membre du Comité central de la FMH et responsable du département Médecine et tarifs ambulatoires commence par une rétrospective:

- Dépôt de la demande d'approbation pour la nouvelle structure tarifaire TARDOC à la mi-juillet 2019

- Échanges écrits entre les partenaires tarifaires et l'OFSP en automne 2019

- À partir de janvier 2020, évaluation par l'OFSP du contenu du dossier déposé

- Trois ateliers d'une demi-journée à l'OFSP sur le TARDOC au printemps 2020

- Négociation d'un concept de neutralité des coûts commun avec curafutura

- SWICA adhère au TARDOC au printemps 2020 (= majorité parmi les assureurs)

- Remise du concept de neutralité des coûts commun au Conseil fédéral en juin 2020

- Le 26 août 2020, l'OFSP a informé curafutura et la FMH par courrier que le TARDOC devait être soumis à une nouvelle évaluation

- Dans son rapport d'évaluation du 19 novembre 2020, l'OFSP arrive à la conclusion que le TARDOC peut matériellement être approuvé, moyennant quelques ajustements

Résumé des principaux points soulevés par le rapport de l'OFSP

1. Modèle de coûts de la prestation médicale (PM): l'OFSP considère que le revenu de référence consigné est trop élevé et qu'il devrait se situer autour de 190000 francs, être calculé à l'échelon des chefs de clinique et s'appuyer sur une durée réglementaire de travail de 11,4 heures par jour. Cela corrigerait fortement le taux de coûts PM à la baisse.

2. Modèles de coûts INFRA et KOREG: la principale critique porte sur l'existence de deux modèles de coûts. Dans KOREG, c'est la représentativité des données qui est remise en question et dans INFRA, les données de référence.

3. Évaluation des conséquences économiques et concept de neutralité des coûts

4. Concept de valeur intrinsèque $\rightarrow$ maintien des droits acquis)

Revenu de référence et horaire de travail annualisé La FMH a soumis ces deux points critiqués à l'appréciation d'experts externes. Les deux expertises ont montré qu'il n'était pas nécessaire d'adapter le modèle de coûts PM, qui a donc été défendu dans la prise de position transmise à l'OFSP. Du point de vue des parte- 


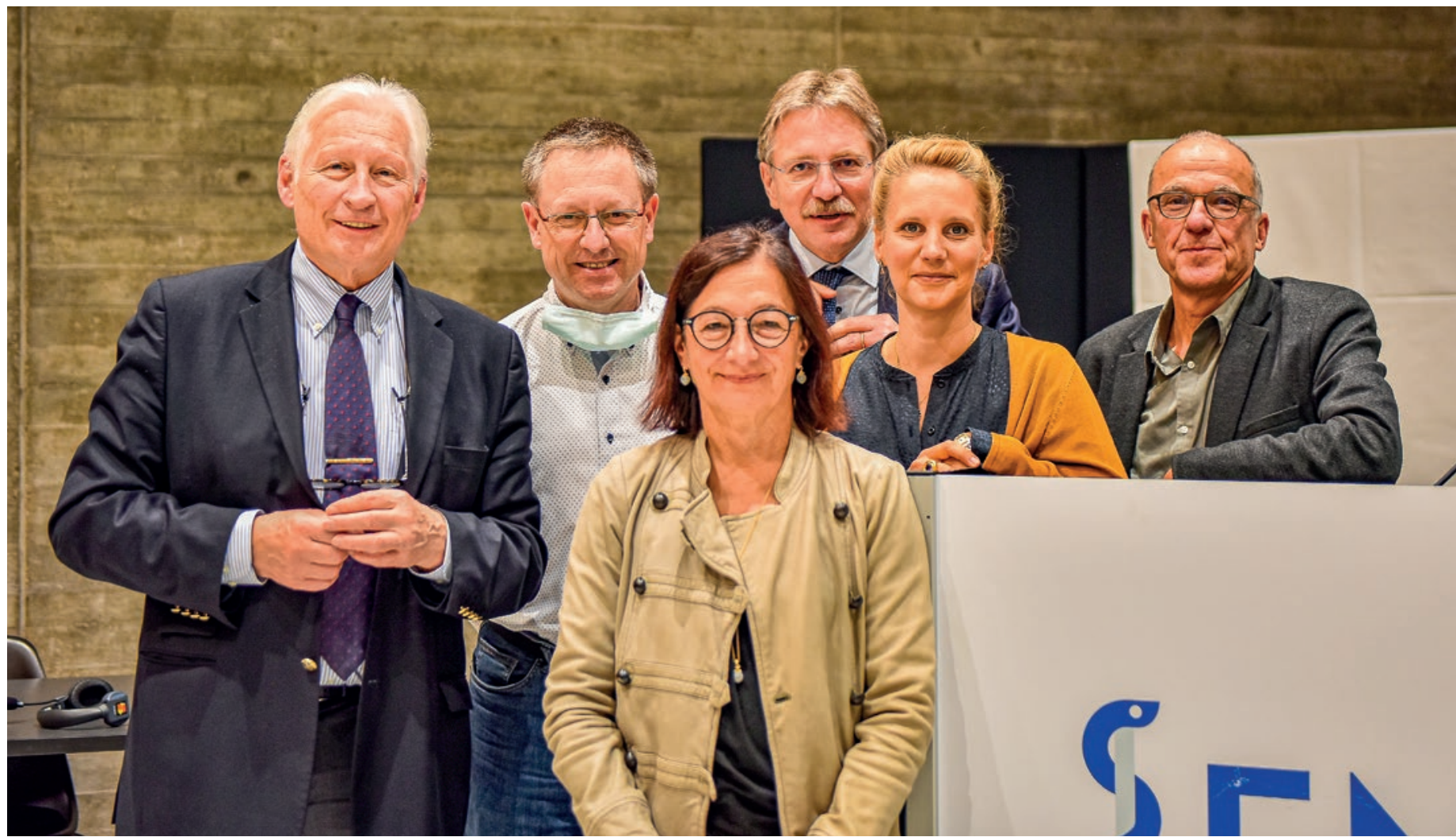

Le Comité central de la FMH, présidé par Yvonne Gilli. De g. à d.: Urs Stoffel, Christoph Bosshard, Yvonne Gilli, Carlos Quinto, Jana Siroka et Alexander Zimmer. Michel Matter n'est pas sur la photo.

naires tarifaires, aucune adaptation du taux de coûts de la PM n'est nécessaire dans le TARDOC.

Adaptation du concept de neutralité des coûts

- L'OFSP a critiqué différents points dans le concept de neutralité des coûts du TARDOC (durée, croissance normative convenue pour 2020 et 2021, marge de tolérance, etc.).

- Seule la phase de mesure et de pilotage a été prolongée de 18 à 30 mois, et ce pilotage portera sur les années 2022 et 2023.

- Pour la marge d'intervention de l'année 2023, il a été prévu +6\%/-2\% par rapport au volume 2021.

- Adaptation du concept conformément à cette prolongation de 12 mois de la phase de mesure et de pilotage.

Adaptations dans le concept de valeur intrinsèque

- curafutura et la FMH ont réduit la durée du maintien des droits acquis de dix à six ans.

- La précision pour faire valoir les droits acquis de «3 ans avant avril 2019» a été modifiée: «3 ans avant l'entrée en vigueur".

curafutura et la FMH ont remis ensemble la version révisée du TARDOC à l'OFSP, le 30 mars 2021.

Le Conseil fédéral a convié tous les partenaires tarifaires de la LAMal à plusieurs tables rondes (y compris une représentation de la Conférence des directrices et directeurs cantonaux de la santé). Une lettre d'intention commune a pu être élaborée et signée.

\section{Informations du Comité central, du Secrétariat général, de l'ISFM et des divisions}

Yvonne Gilli / présidente de la FMH déclare que les informations relatives aux départements peuvent être consultées sur myFMH. Christoph Bosshard et Jana Siroka prennent la parole pour s'exprimer sur leur département respectif.

Art. 58 LAMal Qualité et économicité: informations actuelles

Christoph Bosshard / vice-président de la FMH et responsable du département Données, démographie et qualité précise l'objectif, les tâches et les compétences de la Commission fédérale pour la qualité et les conventions qualité nationales qui résultent de cet article de loi, entré en vigueur le $1^{\text {er }}$ avril 2021.

Il évoque ensuite le contenu général (projet) de ces conventions qualité, qui se base principalement sur les dispositions légales. Pour l'instant, les négociations relatives à une convention-cadre sont en cours avec les 
assureurs santésuisse et curafutura. Une prochaine étape consistera à mettre sur pied un concept qualité (national), dans lequel seront dessinées les exigences concernant le développement de la qualité, les activités dans ce domaine et la manière de la mesurer mais aussi les mesures d'amélioration de la qualité des fournisseurs de prestations (annexes au concept). Il est cependant important que les principales activités qualité soient définies et fixées par les sociétés de discipline. Les organisations représentées à la Chambre médicale seront associées suffisamment tôt au processus décisionnel.

\section{ST Reha 1.0}

Jana Siroka / membre du Comité central de la FMH et responsable du département Médecine et tarifs hospitaliers informe du système tarifaire dans le domaine de la réadaptation (forfaits journaliers liés aux prestations), adopté par le Conseil d'administration de SwissDRG SA. Introduction prévue le $1^{\text {er }}$ janvier 2022.

Dès la phase préparatoire, la FMH et les sociétés de discipline ont collaboré à la définition des prestations de base et des prestations supplémentaires relatives aux différents types de réadaptation. Jana Siroka / membre du Comité central de la FMH et responsable du département Médecine et tarifs hospitaliers précise que ces travaux conséquents ont en grande partie été repris dans la CHOP et la structure tarifaire ST Reha.

Plusieurs points importants soulevés par le corps médical ont été entendus. SwissDRG SA a, par exemple, effectué l'intervention normative demandée, ce qui a permis de lever un incitatif négatif considérable. Par ailleurs, la FMH a réussi à obtenir un délai pour l'utilisation du codage par analogie, permettant ainsi de garantir un meilleur lien entre tarif et prestation.

Pour conclure, Jana Siroka / membre du Comité central de la FMH et responsable du département Médecine et tarifs hospitaliers rappelle que les structures tarifaires ST Reha, SwissDRG et TARPSY sont en évolution permanente, la FMH s'y attèle et le corps médical peut faire valoir son avis, notamment en participant aux procédures de proposition dans le but de perfectionner les tarifs. La FMH apporte son soutien aux sociétés de discipline et aux organisations faîtières dans cette dé- marche. Elle défend par ailleurs le point de vue des médecins dans les différents organes de SwissDRG SA et auprès de l'Office fédéral de la statistique. Elle propose entre autres les priorités vers lesquelles orienter les développements.

\section{Divers}

\subsection{Assemblée des délégués et Chambre médicale: changement de dates}

Yvonne Gilli / présidente de la FMH informe des dates des séances en 2021/2022.

Dates de l'Assemblée des délégués et de la Chambre médicale en 2021

L'Assemblée des délégués prévue le 8 septembre aura lieu le $1^{\text {er }}$ septembre 2021.

La Chambre médicale prévue le 28 octobre aura lieu le 7 octobre 2021.

\section{Dates de la Chambre médicale en 2022}

Jeudi 19 mai 2022

Jeudi 27 octobre 2022

\subsection{Groupe de travail de la FMH sur le change-} ment climatique et la santé: report

Yvonne Gilli / présidente de la FMH informe que l'Assemblée des délégués a décidé de reporter la présentation, la discussion et l'adoption de la stratégie (incluant le budget) du corps médical suisse sur le thème du changement climatique et de la santé. Le sujet sera traité en septembre 2021 à l'Assemblée des délégués et en octobre 2021 à la Chambre médicale.

Yvonne Gilli / présidente de la FMH remercie les délégué-e-s pour leur participation active et constructive et en particulier pour leur soutien et leur patience lors de cette séance virtuelle. Elle remercie également les équipes techniques sur place, les divisions Secrétariat central et Service juridique et toutes celles et ceux qui ont participé à l'organisation et la réalisation de cette Chambre médicale.

Yvonne Gilli / présidente de la FMH clôt la séance et prend congé de l'assemblée. 


\section{Glossaire}

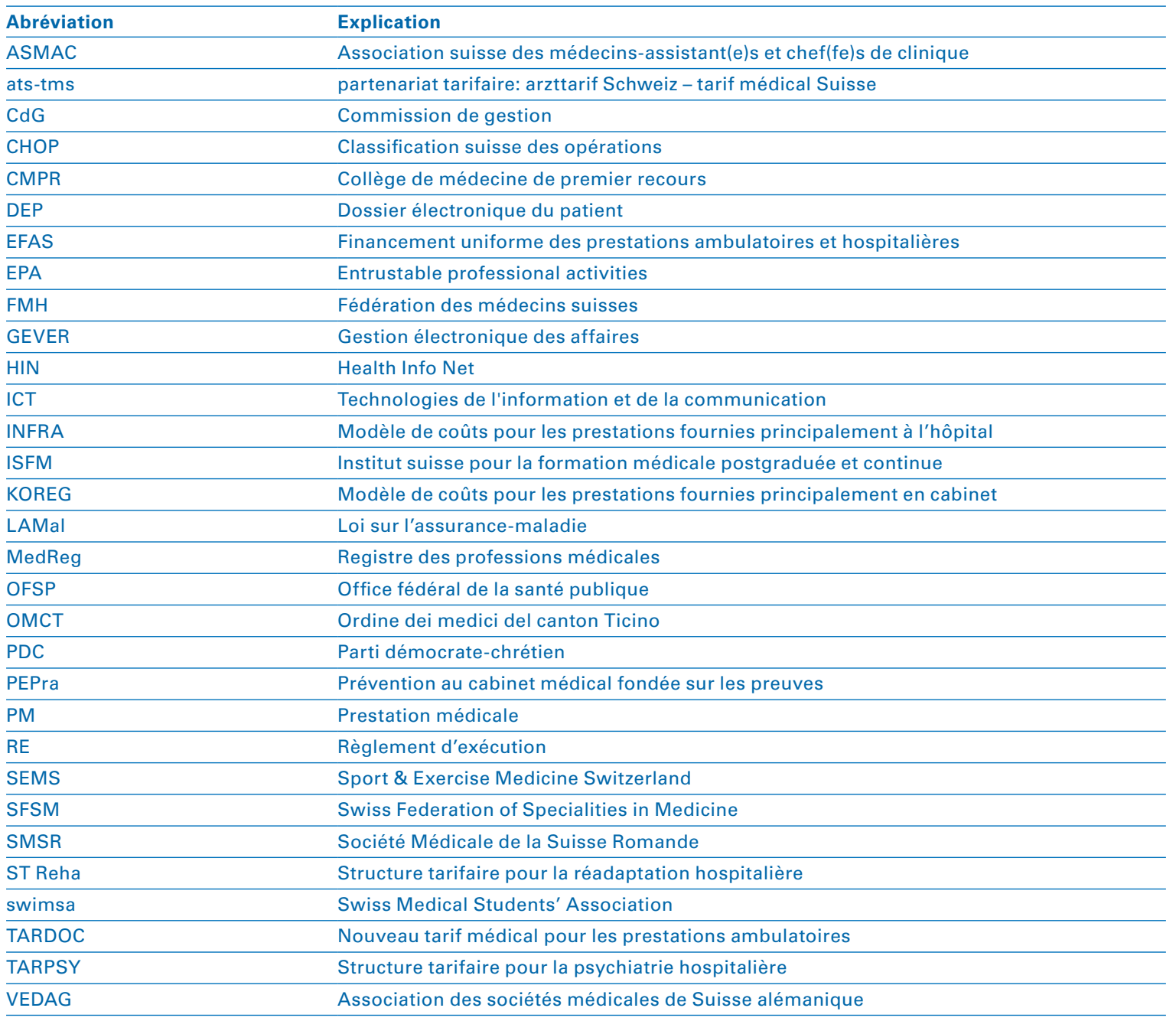

